\title{
Pelvi-perineal flap reconstruction: normal imaging appearances and post-operative complications on cross-sectional imaging
}

\author{
Nyree Griffin • Jeremy Rabouhans • Lee A. Grant • \\ Roy L. H. Ng • David Ross • Paul Roblin • \\ Mark L. George
}

Received: 26 September 2010 /Revised: 3 December 2010 /Accepted: 14 January 2011 /Published online: 2 February 2011

(C) European Society of Radiology 2011

\begin{abstract}
Radical pelvic surgery is often required in patients with advanced, persistent or recurrent gynaecological and anorectal malignancies. In the last decade, pedicled flap reconstructions have been increasingly used for pelvic floor and neovaginal reconstruction, introducing well-vascularised non-irradiated tissue into the wound cavity and hence reducing wound complications. The aim of this pictorial review is to describe the normal post-operative cross-sectional imaging appearances of the most commonly used pelvi-perineal flap reconstructions and to illustrate the complications that may arise at the flap donor and recipient sites.
\end{abstract}

Keywords Surgical flaps · Computed tomography ·

Magnetic resonance imaging $\cdot$ Post-operative

complications $\cdot$ Pelvic exenteration

N. Griffin $(\bowtie) \cdot$ J. Rabouhans

Department of Radiology, Guys' and St Thomas' Hospital,

Westminster Bridge Road,

London, UK SE1 7EH

e-mail: nyree.griffin@gstt.nhs.uk

L. A. Grant

Department of Radiology, Royal Free Hospital,

London, UK

R. L. H. Ng $\cdot$ D. Ross $\cdot$ P. Roblin

Department of Plastic Surgery, Guy's and St Thomas' Hospital,

London, UK

M. L. George

Department of General Surgery, Guy's and St Thomas' Hospital, London, UK

\section{Introduction}

In recent years, radical pelvic surgery has been increasingly undertaken in patients with locally advanced, persistent or recurrent gynaecological and anorectal malignancies. Surgery is carried out either as a curative or palliative procedure and includes an extended abdominoperineal excision (APE) and possibly an anterior, posterior or total pelvic exenteration (TPE). These radical surgical techniques are associated with high morbidity, with the risk of both local and more generalised complications. A wide cutaneous defect and large dead space within the pelvis increases the risk of postoperative perineal complications that occur in up to $25-60 \%$ of patients [1,2]. Local complications include wound dehiscence, abscess or haematoma formation, fistula or sinus formation, delayed wound healing and, rarely, a perineal hernia. The frequent use of neoadjuvant (chemo-)radiotherapy in order to reduce local recurrence rates also contributes to delayed wound healing, due to ischaemic hypoxia from vessel thrombosis and impaired fibroblast proliferation [3-5]. In an effort to overcome these problems, pedicled flaps (in the form of muscle, myocutaneous or fasciocutaneous flaps) have been increasingly employed. This method introduces well-vascularised non-irradiated tissue into the wound cavity and can be used for pelvic floor and, in the case of female patients, neovaginal reconstruction.

Cross-sectional imaging is a valuable tool in the postoperative evaluation of patients with pelvi-perineal flap reconstruction. Both computed tomography (CT) and magnetic resonance imaging (MRI) assist in the detection of complications related to the flap donor and recipient sites as well as in the recognition of tumour recurrence. With several flap techniques now used, it is essential that the radiologist 
can recognise normal post-operative imaging appearances in order to appreciate any complications that may arise. To date, there has been very little literature on the cross-sectional imaging appearances of such patients $[6,7]$.

The purpose of this paper is twofold: (1) to describe the three most commonly employed surgical techniques used for pelvi-perineal flap reconstruction at our centre-i.e. the vertical rectus abdominis myocutaneous (VRAM) flap, the gracilis flap and the inferior gluteal artery perforator (IGAP) flap - and their normal post-operative appearances on CT and MRI, and (2) to illustrate the post-operative complications related to both the flap donor and recipient sites.

\section{Surgical technique and normal cross-sectional imaging appearances}

\section{The VRAM flap}

A right-sided VRAM flap is usually created, as patients normally require a left-sided colostomy. Rarely, a left-sided VRAM flap is required if there has been previous scarring or a stoma is present on the right hand side. The flap consists of a 5- to $10-\mathrm{cm}$ wide skin paddle designed vertically above the right rectus abdominis muscle. Initially, the anterior rectus sheath fascia is incised and the rectus muscle and the overlying soft tissue is elevated away from the posterior rectus sheath. The pelvic insertion of the muscle is left intact. The superior epigastric vessels are ligated. The deep inferior epigastric artery and vein are isolated to mobilise the pedicle. The flap is then rotated medially and passed into the pelvis to be inserted into the defect created by the resection, with the distal end of the flap lying posteriorly. The skin paddle of the VRAM flap is used to reconstruct the posterior vaginal wall when indicated. Following resection of the primary tumour and formation of the contralateral colostomy, the abdominal wall donor site is closed with a prosthetic mesh.

The VRAM flap was first described by Shukla and Hughes in 1984 [8] and popularised by Tobin et al. in 1988 [9]. The advantages of this flap are that it provides bulkier tissue compared with the other flaps, in order to fill the large dead space. The flap has a wide arc of rotation. The scar for the flap can be incorporated into the laparotomy scar for excision of the primary tumour. The flap prevents small bowel herniation into the pelvis, and thus reduces the risk of adhesions and small bowel fistulas; the latter complication is reduced from 16 to $5 \%[10,11]$. One disadvantage of the VRAM flap is that it may result in an excessive amount of tissue within the perineal area that cannot be easily shaped.

On cross-sectional imaging, the VRAM flap is the easiest of the three flaps to recognise. The donor site (usually the right side of the anterior abdominal wall) is thinner than the contralateral side due to mobilisation of the rectus flap (Figs. 1, 2). This flap reconstruction is usually performed in conjunction with an APE, with the creation of a colostomy in the left iliac fossa. Within the pelvis, the flap is seen orientated obliquely, extending from the linea alba (usually to the right of the midline), posteriorly to the concavity of the sacrum (Figs. 2, 3). In the case of an anterior exenteration, the flap extends posteriorly to the peri-rectal fascia. The vascular pedicle can often be seen, especially following administration of intravenous contrast. It will arise from the external iliac vessels and turn posteromedially into the pelvis lateral to the flap (Fig. 2). The bulk of the flap is of fat density corresponding to the subcutaneous fat, with a strip of muscle density noted within it, corresponding to the rectus abdominis muscle. With time, this muscle component can undergo atrophy due to denervation [6], eventually appearing reduced in size, and showing fatty infiltration. Atrophy may lead to an increased signal intensity of the muscle component on both T1- and T2-weighted MRI. If a neovagina is present, it is formed from the flap skin, which is completely encircled by subcutaneous fat. Air within the neovagina is a normal finding. Pre-sacral soft tissue thickening may also be a normal post-operative finding and should remain stable or become less prominent on subsequent imaging.

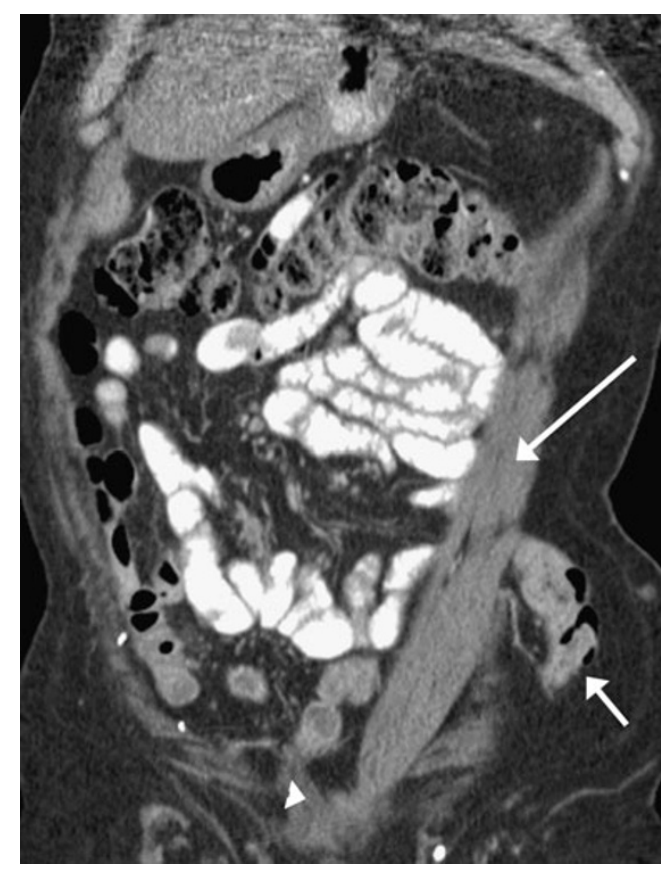

Fig. 1 Normal VRAM flap donor site appearances on CT. Coronal reformat contrast-enhanced CT (CECT), in a patient who underwent APE and right VRAM flap reconstruction, shows colostomy in the left iliac fossa (short arrow) in association with a normal left rectus abdominis muscle (long arrow). The right rectus abdominis muscle flap is seen passing into the pelvis (arrowhead) 


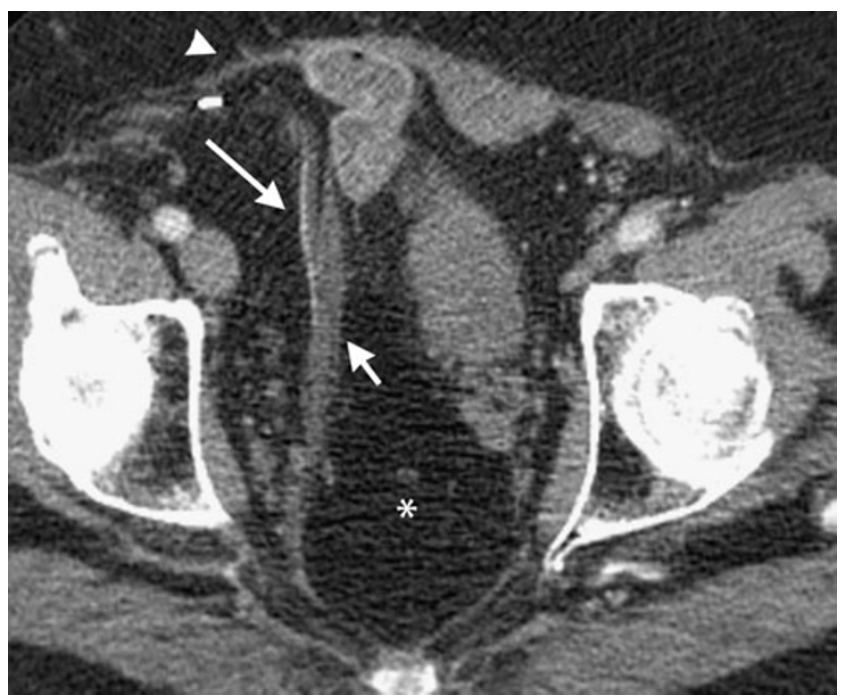

Fig. 2 Normal VRAM flap appearances on CT. Axial CECT of the abdomen shows absent right rectus abdominis muscle in keeping with the donor site (arrowhead). The flap passes posteriorly from the right linea alba into the pelvis. The fat density within the pelvis corresponds to the subcutaneous fat component of the flap (asterisk), whilst the strip of muscle density seen in the right hemipelvis corresponds to the donor rectus abdominis muscle (short arrow). Note the inferior epigastric artery is seen as an enhancing linear structure arising from the external iliac vessels lateral to the muscle component of the flap (long arrow)

\section{The gracilis myocutaneous flap}

The gracilis muscle is a slim superficial muscle on the medial aspect of the thigh (Fig. 4), originating from the symphysis pubis and superior pubic ramus and inserting into the medial aspect of the proximal tibia. The flap is designed by drawing a line from the pubic tubercle to the medial condyle of the distal femur, and a skin paddle is designed to overlie this. Its size is tailored to that of the surgical defect. An elliptically shaped skin paddle is outlined, and the marked skin paddle is incised to the level of the gracilis fascia. The gracilis muscle is identified beneath this and transected just proximal to its distal insertion. The flap is then raised from distal to proximal, retaining the perforating vessels from the muscle to the skin. A subcutaneous tunnel is created proximally in the thigh to gain access to the surgical defect. The flap is rotated on its vascular pedicle (which is usually a branch of the medial femoral circumflex artery) and inserted into the surgical defect. As with the VRAM flap, the skin paddle is used to reconstruct the posterior vaginal wall when necessary.

The gracilis myocutaneous flap was first described for perineal and neo-vaginal reconstruction by $\mathrm{McCraw}$ et al. in
Fig. 3 Normal VRAM flap appearances on MRI. Sagittal T2-weighted images show (a) a strip of muscle signal intensity extending posteriorly from the anterior abdominal wall, corresponding to the rectus abdominis flap (arrow) and (b) muscle flap terminating presacrally (arrow) with the subcutaneous fat component seen filling the pelvis and perineal defect (asterisk). c Coronal T2weighted image of the same patient shows the muscle flap passing into the right hemipelvis (arrow) with subcutaneous fat component of the flap again well demonstrated (asterisk)
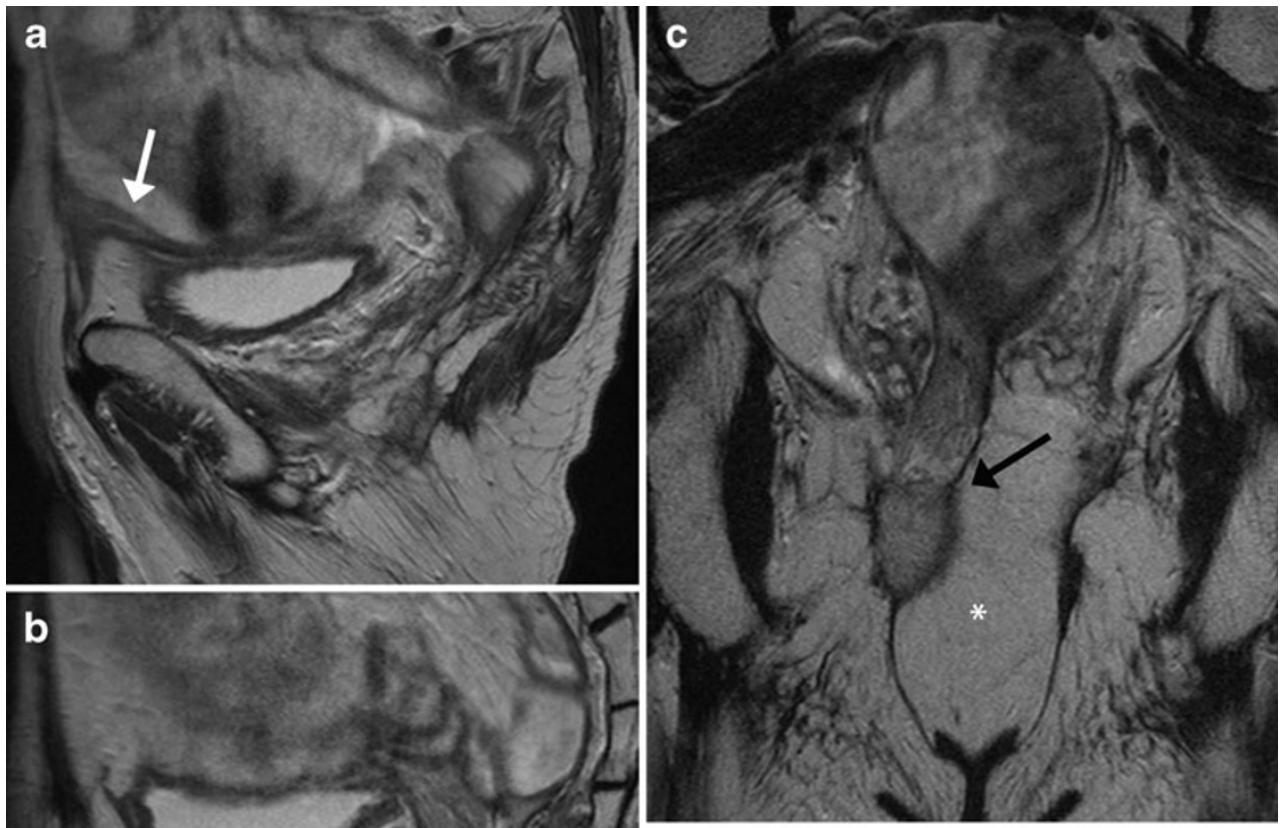


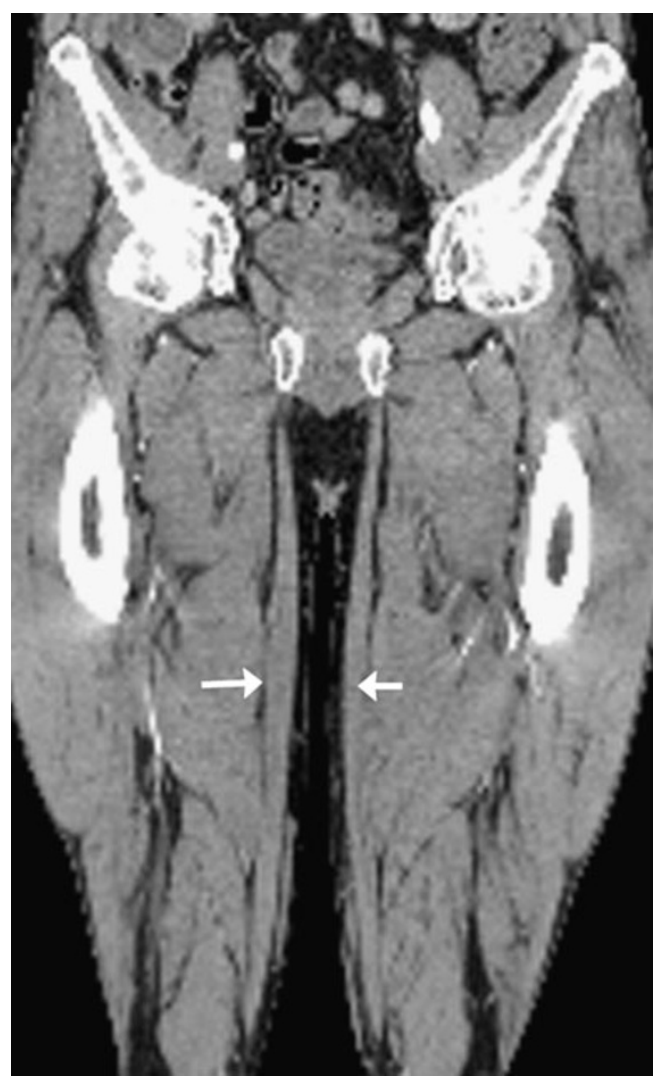

Fig. 4 Normal anatomy of the gracilis muscle. Coronal reformat CECT showing the normal anatomy of the gracilis muscle in the medial aspect of the thigh (arrows). It arises from the symphysis pubis and superior pubic ramus to insert into the medial aspect of the proximal tibia

1976 [12]. Its use may be limited due to an inferiorly sited dominant vascular pedicle, with a distal vascular origin resulting in a restricted arc of rotation. Donor site scars are cosmetically conspicuous.

At our centre, the gracilis myocutaneous flap is used in patients who require a double stoma following total pelvic exenteration. A bilateral gracilis flap harvest is performed when a total vaginectomy has been carried out and a neovaginal reconstruction is required.

On cross-sectional imaging the normally sited gracilis muscle, along the medial aspect of the thigh, is not visualised due to mobilisation of the gracilis myocutaneous flap. Surgical clips or inflammatory change may denote the donor site (Fig. 5). Instead, the transposed flap is seen within the perineum, with the muscle belly situated laterally and the subcutaneous fat component of the flap seen medially (Fig. 6a-c). Bilateral flaps will give a symmetrical appearance on either side of the midline. The skin of the flap can form the vaginal lining when a vaginal reconstruction is carried out. This flap is usually performed in the context of a total pelvic exenteration (comprising a cystectomy, APE, removal of the urogenital organs, and pelvic lymphadenectomy). The patient will have both an ileal conduit for urinary diversion (within the right iliac fossa) and a colostomy (within the left iliac fossa).

\section{The inferior gluteal artery perforator flap (IGAP) flap}

This is a fasciocutaneous flap based on the inferior gluteal artery perforating vessels. The dimensions of the flap are determined by the surface area to be covered. The flap is elevated from the underlying gluteus muscle, with the inferior gluteal and sciatic nerves lying deep to the plane of dissection. The inferior gluteal artery perforator is identified and dissected throughout its length, allowing the flap enough freedom to be advanced into the defect. Bilateral flaps are raised in nearly all of our patients having this form of reconstruction. One flap is placed deep into the pelvis to control the dead space and the second is then brought across the midline to cover the perineal defect.

The IGAP fasciocutaneous flap is a modification of the musculocutaneous gluteal flap, originally described in 1978 by Shaw and Futrell for the closure of perineal sinuses [13]. Assessment of the inferior gluteal artery perforators prior to surgery has been described using CT angiography and Doppler ultrasound [14]. The use of the gluteus maximus muscle is avoided - this is advantageous as it is important for gait and is thus best preserved [15].

The IGAP flap is the most difficult of the three flaps to appreciate on cross-sectional imaging. At the donor site, the subcutaneous fat of the gluteal region on one or both sides may be reduced in bulk compared with any preoperative imaging (Fig. 7). Yet again, surgical clips or stranding of

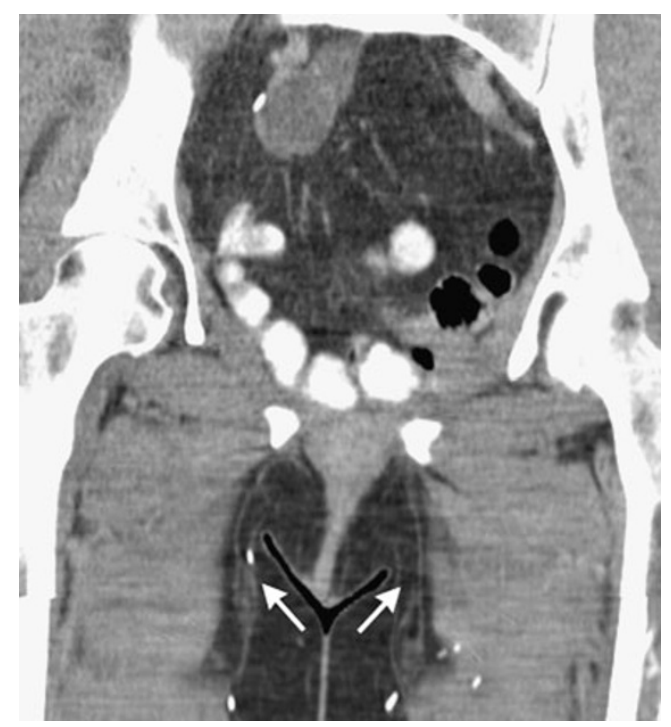

Fig. 5 Normal gracilis flap donor site appearances on CT. Coronal CECT showing absence of the normally sited gracilis muscle in the upper thigh (arrows) in a patient with bilateral gracilis flap reconstruction. Surgical clips and fat stranding is noted at the donor sites 


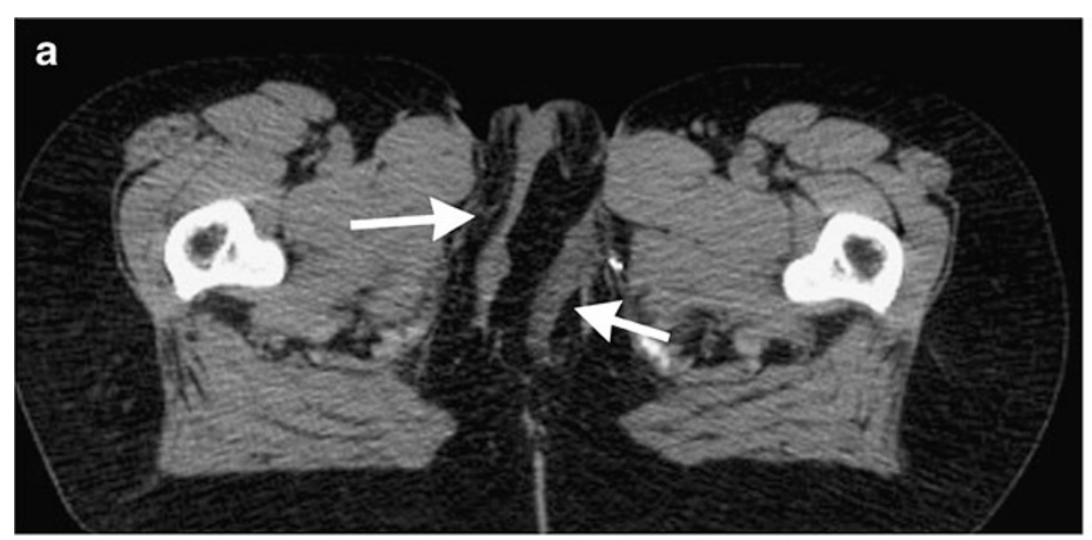

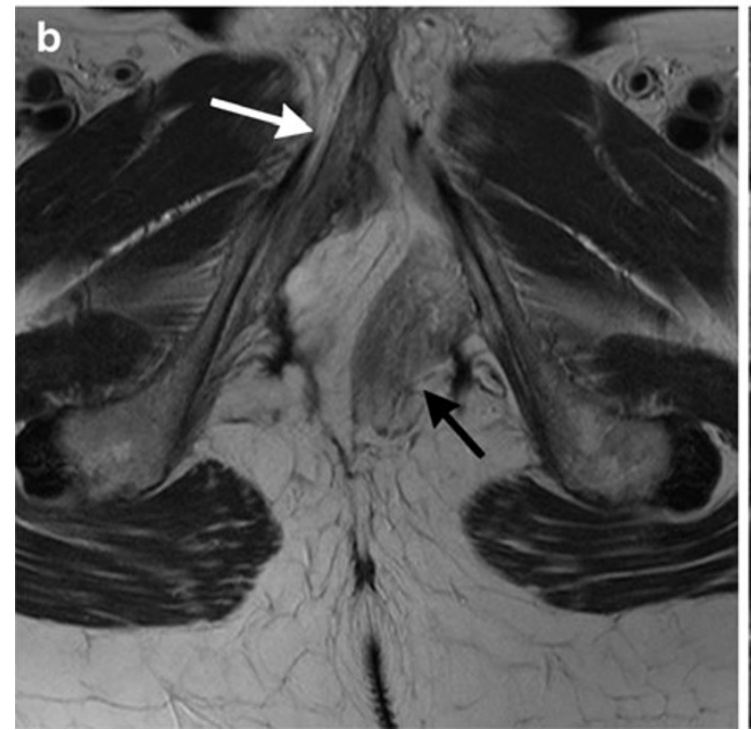

Fig. 6 Normal gracilis flap recipient site appearances on CT and MRI. Patient has had a total pelvic exenteration and bilateral gracilis flap reconstruction: (a) axial CT shows bilateral gracilis flaps in the

the subcutaneous fat may denote the donor site. The pelvic floor reconstruction will be seen chiefly of fat density. As with the other flaps, the skin of the flap is used to reconstruct the lining of the neovagina with gas sometimes seen internally.

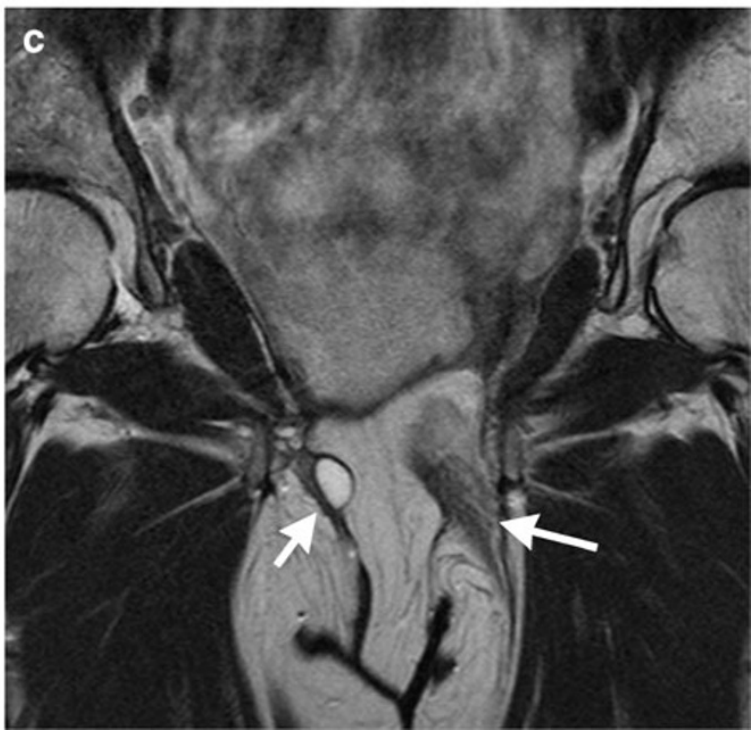

perineum as laterally placed strips of muscle with central fat density; (b) axial and (c) corresponding coronal T2-weighted MRI shows bilateral gracilis flaps (arrows) with small seroma on the right-sided flap

\section{Complications related to the flap donor site on cross-sectional imaging}

Donor site complications that have been described include wound infection, skin edge necrosis, wound dehiscence,
Fig. 7 Normal inferior gluteal artery perforator (IGAP) flap appearances on CECT in a patient following APE and IGAP flap reconstruction for rectal cancer. Axial CECT shows surgical clips and stranding/loss of volume of the subcutaneous fat overlying the gluteus maximus bilaterally (arrows), corresponding to the donor sites. The transposed flap is seen as central fat density (asterisk)

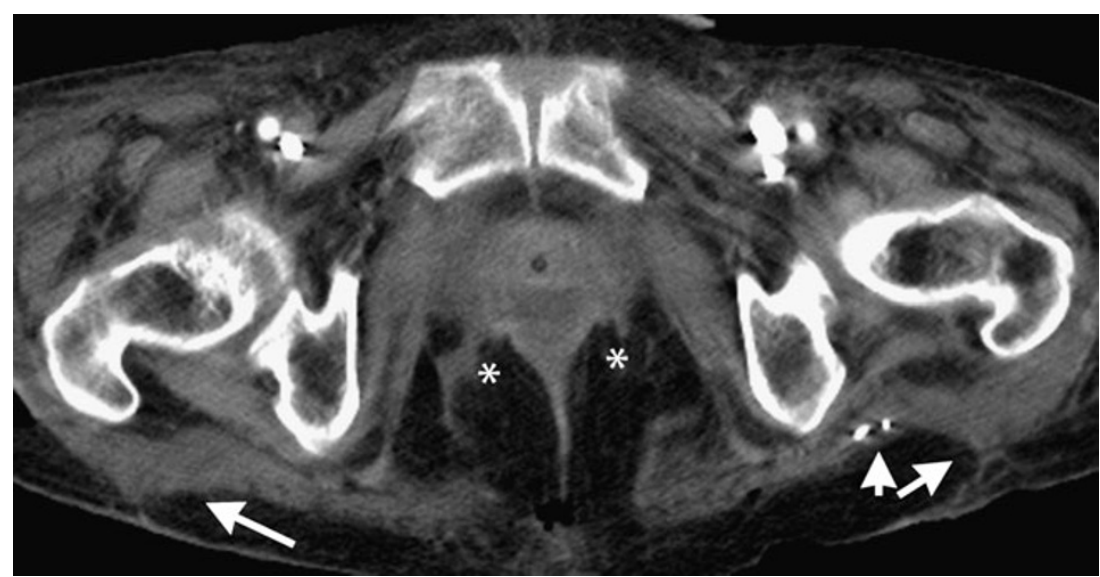




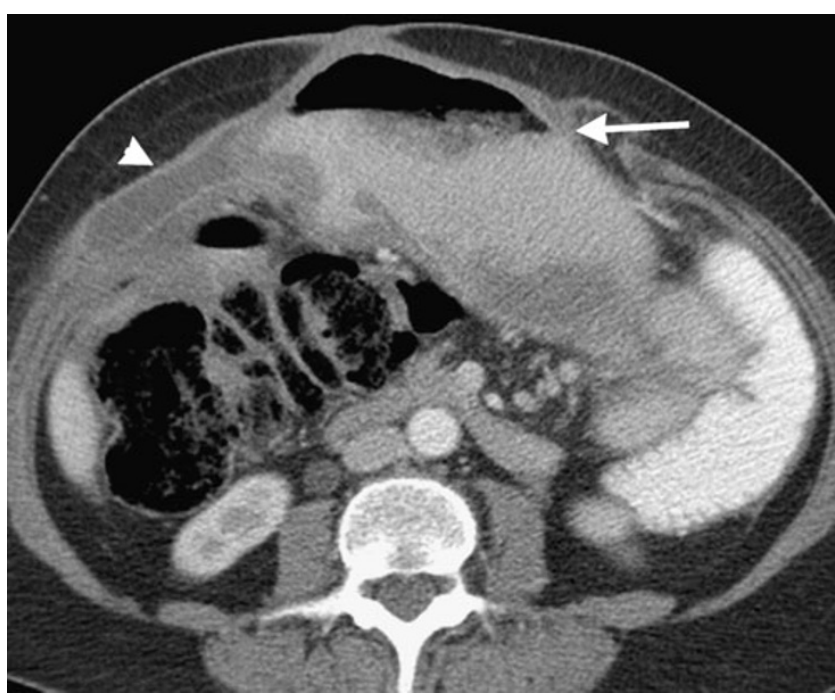

Fig. 8 Donor site complication in a patient who underwent APE and VRAM flap reconstruction. CECT showing stomach prolapsing into an incisional hernia (arrow) associated with a small seroma at the donor site (arrowhead)

seromas (Fig. 8) and incisional hernias (Figs. 8, 9) [16, 17]. Donor site seromas appear as small, thin-walled fluid collections of varying sizes associated with the resected anterior abdominal wall that usually resolve on subsequent imaging. The VRAM flap leaves a weakened anterior abdominal wall, and therefore predisposes to abdominal wall dehiscence and incisional hernia formation in approximately $5 \%$ of patients [18]. Wound dehiscence is made more likely if there is increased wound tension (e.g. when a large defect has to be closed) or in the presence of a wound infection. In order to reduce the incidence of incisional

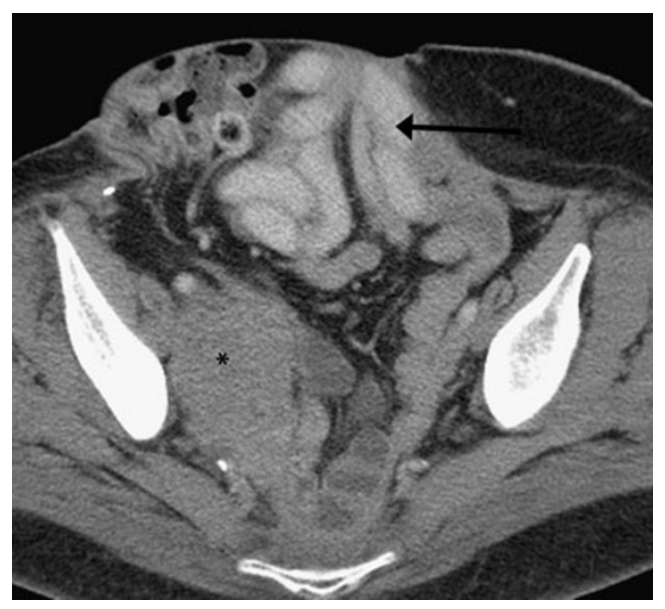

Fig. 9 Donor site complication in a patient who underwent APE and VRAM flap reconstruction. CECT showing a large incisional hernia containing loops of small and large bowel (arrow) following previous abdominal wound dehiscence in a patient with right VRAM flap reconstruction; this patient also had a recurrent tumour seen as enhancing soft tissue along the right pelvic side wall (asterisk)

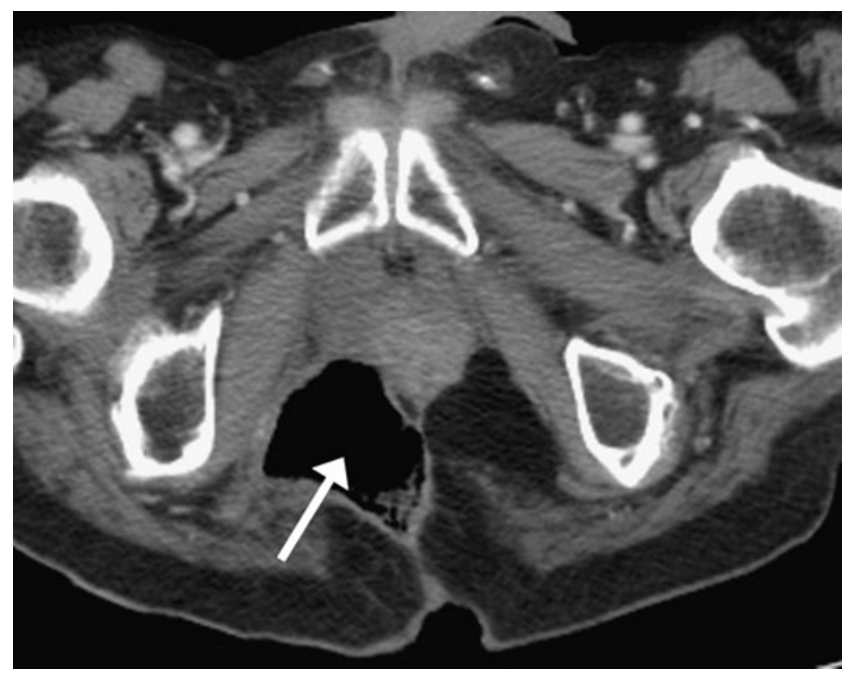

Fig. 10 Recipient site complication. CECT showing an example of a flap breakdown (arrow) in a patient following TPE and gracilis flap reconstruction

hernias, the posterior rectus sheath is left intact at the donor site. A mesh is used to repair the anterior rectus sheath and strengthen the abdominal wall.

\section{Complications related to the flap recipient site on cross-sectional imaging}

Flap recipient site complications that have been reported include flap necrosis, perineal or pelvic collections or abscesses, perineal hernia and fistula formation $[16,19$, 20]. Total flap loss may result from an arterial or venous thrombosis or poor arterial inflow due to excess traction or kinking of the pedicle and requires full debridement and

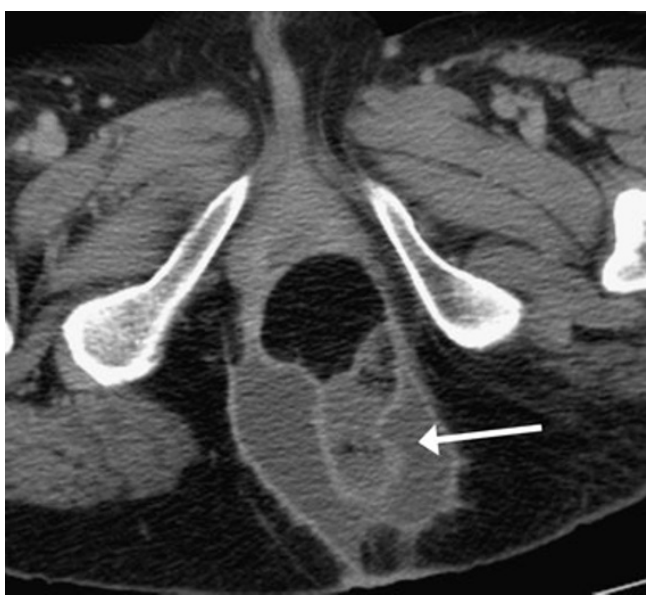

Fig. 11 Recipient site complication. CECT showing a perineal rim enhancing fluid collection (arrow) associated with a VRAM flap reconstruction for rectal cancer 


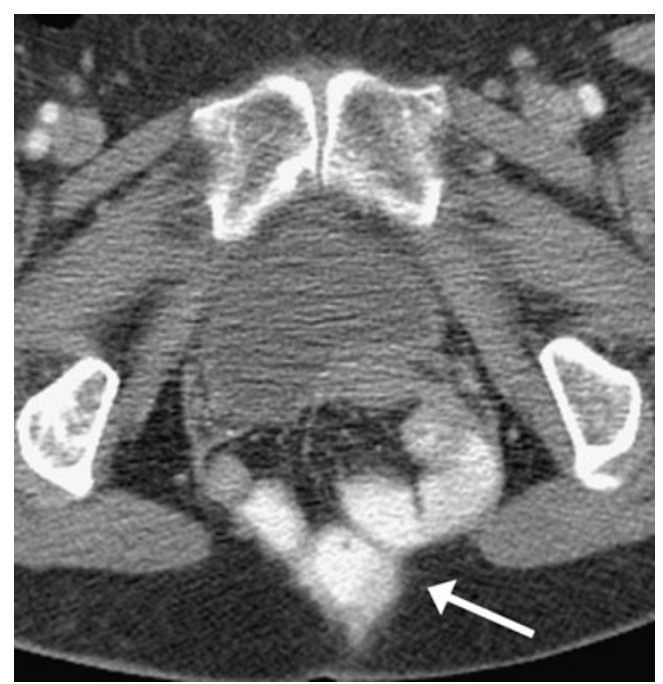

Fig. 12 Recipient site complication. CECT showing an example of a perineal hernia (arrow) with prolapsing loops of small bowel into the perineum in a patient following APE and IGAP flap reconstruction for rectal cancer
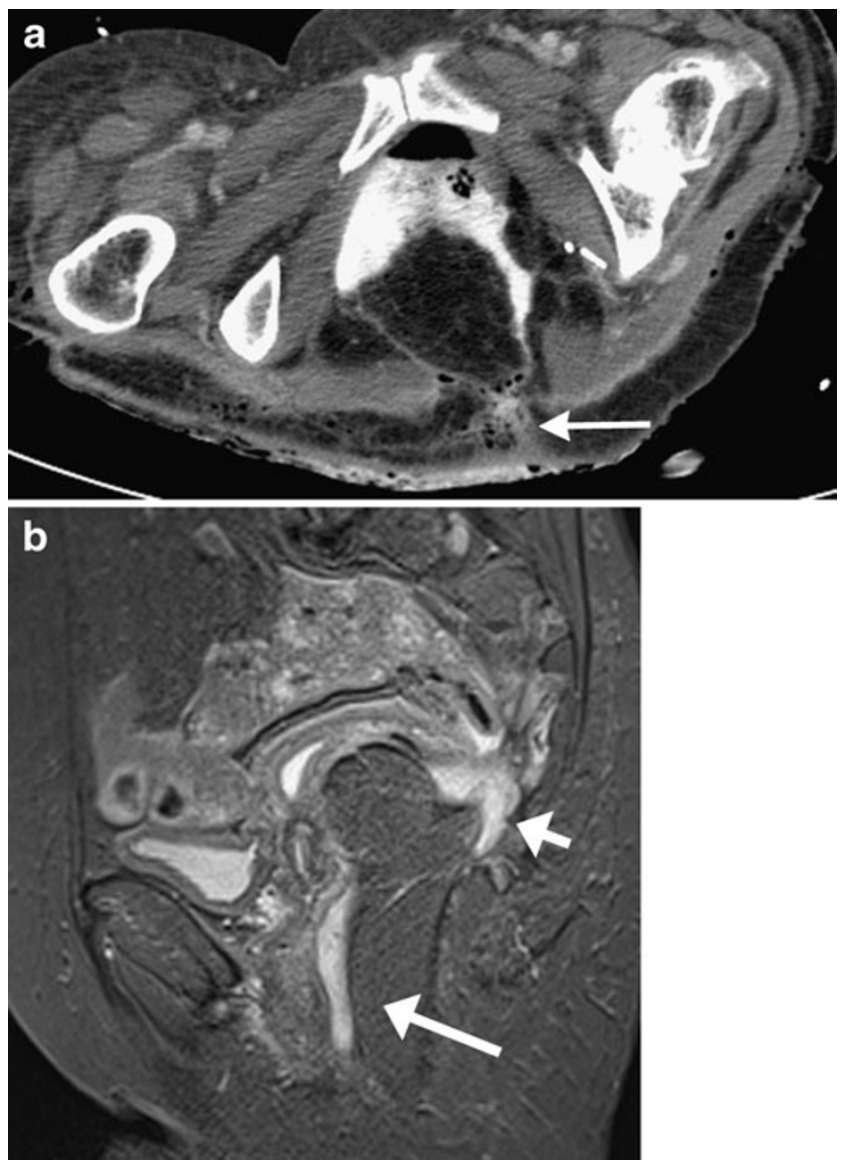

Fig. 13 Recipient site complications. a CECT showing an enterocutaneous fistula in a patient following APE, anterior exenteration and IGAP flap reconstruction - oral contrast is seen tracking to the skin surface and pockets of gas are noted in the soft tissues (arrow). b Sagittal STIR MRI in a different patient who underwent APE and VRAM flap reconstruction for rectal cancer shows the presence of high signal perineal sinus tracts around the flap (arrows)

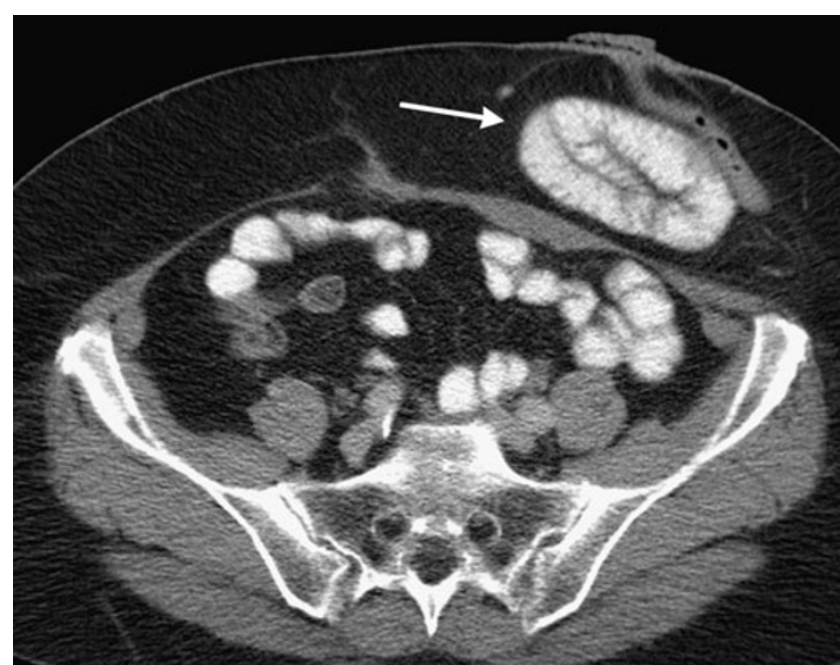

Fig. 14 Other post-operative complications. CECT showing a parastomal hernia containing loops of small bowel (arrow), herniating alongside the colostomy in a patient who underwent APE, VRAM flap reconstruction and stoma formation

reconstruction. Partial flap loss may sometimes be managed by debridement alone. Assessment of impaired perfusion and early flap failure can be made with dynamic contrastenhanced (DCE) MRI, although this is not standard practice. It has been shown that there is a decreased enhancement curve within a failing flap on DCE-MRI compared with that of the surrounding tissues [21]. On cross-sectional imaging, flap loss is manifest by either minor loss of skin and subcutaneous tissue from the margins of the flap to complete loss of the whole flap, resulting in a large defect at the recipient site (Fig. 10). The VRAM flap is more reliable than the gracilis flap, with a total flap loss rate of less than 5\% [22]. There is a higher

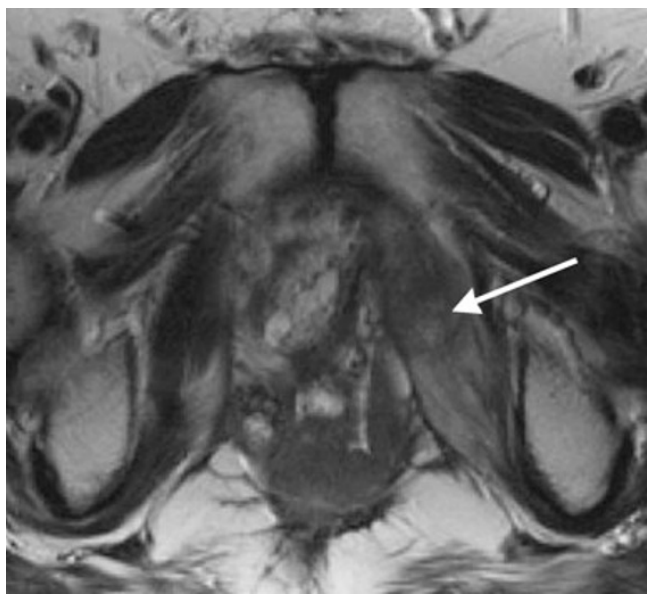

Fig. 15 Axial T2-weighted MRI in a patient following total pelvic exenteration (TPE) and gracilis flap reconstruction for rectal cancer shows intermediate signal intensity in an expanded left obturator internus (arrow), which on biopsy was confirmed to be tumour recurrence 
rate of flap loss in patients with prior stomas on the side of the rectus flap and in patients with multiple previous abdominal incisions due to disruption of part of the deep inferior epigastric artery [23]. Flap necrosis and loss occurs in up to $20 \%$ of patients with gracilis flap reconstructions [12]. The site of the perforating vessels from the muscle to the overlying skin paddle can be unpredictable and the correct positioning of the skin paddle (especially in patients who are obese with sagging inner skin of the thigh) may be inaccurate. Another possible explanation for increased flap loss with the gracilis flap is compression of the vascular pedicle as the flap is rotated through the subfascial tunnel under the pubic ramus. In comparison, the VRAM flap has a larger vascular pedicle and is rotated medially into the pelvis without directly crossing any bony structures. Other risk factors that may compromise the blood supply include smoking, other co-morbidities associated with small vessel disease, or prior chemoradiation.

Pelvic and perineal abscess collections have been shown to be significantly less in patients undergoing a VRAM flap reconstruction compared with a primary closure [19] (Fig. 11) [24]. Perineal hernias are a recognised complication due to weakness of the pelvic floor, usually seen as loops of prolapsing small bowel into the perineum (Fig. 12). The risk of bowel fistula formation has been reported in up to $5 \%$ of patients with flap reconstructions following exenteration [25]. It is more likely if there is underlying sepsis or iatrogenic injury. Entero-perineal, entero-vaginal and peritoneo-perioneal fistulas may occur, as can chronic perineal sinuses from necrotic flaps. Fistulas are best imaged using $\mathrm{CT}$ with oral contrast (if an enteric fistula is suspected) or with a pelvic MRI with short tau inversion recovery (STIR) sequence (Fig. 13a, b). Fistulas are either treated conservatively or by surgical resection.

\section{Other post-operative complications}

Parastomal hernias (Fig. 14) are a recognised complication with usually part of the small or large bowel herniating out alongside the colostomy.

Although not strictly a post-operative complication, recurrent tumour (Figs. 9, 15) is just as likely to occur in flap reconstruction as with primary surgical closure. Tumour recurrence is not uncommon and early recognition is very important since re-operation after total exenteration is extremely difficult. It is more likely in patients with positive resection margins. Clearly, the development of a new pelvic soft tissue mass on serial post-operative imaging is suggestive of a recurrence. MRI, with its better contrast resolution compared with $\mathrm{CT}$, allows for easier detection of recurrent tumour, with an increased signal on T2-weighted imaging noted within the lesion. Following intravenous contrast administration, recurrent tumour usually enhances. Tumour recurrence should be confirmed histologically with biopsy.

\section{Conclusion}

Perineal flap reconstructions are presently performed in tertiary referral centres with a colorectal surgeon performing the APE and/or pelvic exenteration and a plastic surgeon carrying out the flap procedure. Cross-sectional imaging has proved useful in the follow-up of such patients, and allows for the detection of early and late post operative complications related to both flap donor and recipient sites. As CT is more widely available and is quicker to perform, most follow-up imaging for these patients is with this modality. However, MRI (with its improved contrast resolution) is a useful adjunct, especially in the assessment of possible pelvic recurrence. The reporting radiologist needs to have an awareness of the normal post-operative imaging appearances of perineal flap reconstructions in order to appreciate the development of early and late complications. With the increasing use of neoadjuvant chemoradiotherapy, primary flap reconstructions are likely to be increasingly adopted in preference to primary closure in patients requiring radical pelvic surgery. It is hoped that this review has provided the reader with a greater insight into the cross-sectional imaging appearances and potential complications of the most common types of perineal flap reconstruction presently used for this purpose.

\section{References}

1. Touran T, Frost DB, O'Connel TX (1990) Sacral resection: operative technique and outcome. Arch Surg 25:911-913

2. Wydra D, Emerich J, Sawicki S, Ciach K, Marciniak A (2006) Major complications following exenteration in cases of pelvic malignancy: a 10-year experience. World J Gastroenterol 12:1115-1119

3. Klebanoff SJ (1980) Oxygen metabolism and the toxic properties of phagocytes. Ann Intern Med 93:480-489

4. Rudolph R, Vande Berg J, Schneider JA, Fisher JC, Poolman WL (1988) Slowed growth of cultured fibroblasts from human radiation wounds. Plast Reconstr Surg 82:669-677

5. Krueger WW, Goepfert H, Romsdahl M, Herson J, Withers RH, Jesse RH (1978) Fibroblast implantation enhances wound healing as indicated by breaking strength determinations. Otolaryngology 86:ORL-804-11

6. Epstein DM, Arger PH, LaRossa D, Mintz MC, Coleman BG (1987) CT evaluation of gracilis myocutaneous vaginal reconstruction after pelvic exenteration. AJR Am J Roentgenol 148:1143-1146

7. Willing SJ, Pursell SH, Koch SR, Tobin GR (1991) Vaginal reconstruction with rectus abdominis myocutaneous flap: cT findings. AJR Am J Roentgenol 156:1001-1004

8. Shukla HS, Hughes LE (1984) The rectus abdominis flap for perineal wounds. Ann R Coll Surg Engl 66:337-339 
9. Tobin GR, Day TG (1988) Vaginal and pelvic reconstruction with distally based rectus abdominis myocutaneous flaps. Plast Reconstr Surg 81:62-73

10. Rutledge FN, Smith JP, Wharton JT, O'Quinn AG (1977) Pelvic exenteration: Analysis of 296 patients. Am J Obstet Gynecol 129:881-892

11. Miller B, Morris M, Gerhenson DM, Levenback CL, Burke TW (1995) Intestinal fistulae formation following pelvic exenteration: a review of the University of Texas M.D. Anderson Cancer Center experience, 1957-1990. Gynecol Oncol $56: 207-210$

12. McCraw JB, Massey FM, Shanklin KD, Horton CE (1976) Vaginal reconstruction with gracilis myocutaneous flaps. Plast Reconstr Surg 58:176-183

13. Shaw A, Futrell JW (1978) Cure of chronic perineal sinus with gluteus maximus flap. Surg Gynecol Obstet 147:417420

14. Wagstaff MJ, Rozen WM, Whitaker IS, Enajat M, Audolfsson T, Acosta R (2009) Perineal and posterior vaginal wall reconstruction with superior and inferior gluteal artery perforator flaps. Microsurgery 29:626-629

15. Ramirez OM, Hurwitz DJ, Futrell JW (1984) The expansive gluteus maximus flap. Plast Reconstr Surg 74:757-770

16. Jurado M, Bazan A, Elejabeitia J, Paloma V, Martinez-Monge R, Alcazar JL (2000) Primary vaginal and pelvic floor reconstruction at the time of pelvic exenteration: a study of morbidity. Gynecol Oncol 77:293-297

17. Houvenaeghel G, Ghouti L, Moutardier V, Buttarelli M, Lelong B, Delpero JR (2005) Rectus abdominis myocutaneous flap in radical oncopelvic surgery: a safe and useful procedure. Eur J Surg Oncol Dec 31:1185-1190

18. Nisar PJ, Scott HJ (2009) Myocutaneous flap reconstruction of the pelvis after abdominoperineal excision. Colorectal Dis 11:806-816

19. Butler CE, Gundeslioglu AO, Rodriguez-Bigas MA (2008) Outcomes of immediate vertical rectus abdominis myocutaneous flap reconstruction for irradiated abdominoperineal resection defects. J Am Coll Surg 206:694-703

20. Parsons JK, Tufaro A, Chang B, Schoenberg MP (2003) Rectus abdominis vaginoplasty after anterior exenteration for urologic malignancy. Urology 61:1249-1252

21. Prantl L, Fellner C, Jung M (2010) Evaluation of free flap perfusion with dynamic contrast enhanced magnetic resonance imaging. Plast Reconstr Surg 126:100e-101e

22. Casey WJ III, Tran NV, Petty PM, Stulak JM, Woods JE (2004) A comparison of 99 consecutive vaginal reconstructions: An outcome study. Ann Plast Surg 52:27-30

23. Smith HO, Genesen ML, Runowicz CD, Goldberg GL (1998) The rectus abdominis flap: modifications, complications and sexual function. Cancer 83:510-520

24. Shibata D, Hyland W, Busse P, Kim HK, Sentovich SM, Steele G et al (1999) Immediate reconstruction of the perineal wound with gracilis muscle flaps following abdominoperineal resection and intraoperative radiation therapy for recurrent carcinoma of the rectum. Ann Surg Oncol 6:33-37

25. Turrini O, Guiramand J, Moutardier V, Viret F, Mokart D, Madroszyk A et al (2006) Perineal small bowel fistula after pelvic exenteration for cancer: technical guidelines for perineal fistula. Ann Surg Oncol 13:1622-1626 